\title{
INFLUENCE OF EPHEMERIS UNCERTAINTIES AND RELATIVITY MODELING ON LUNAR LASER RANGING DATA ANALYSIS
}

\author{
Wenjing Jin Huaguan Xn \\ Shanghai Observatory, Shanghai 200030, China. \\ Zhaoming Nie Jinling Li \\ Yunna.n Observatory, P.O. Box 110 ,Kumming,China
}

\begin{abstract}
In this paper anthors pointed out that the root menn square of post-residual is two or three times larger than that of normal points. It is mainly due to the ephemeris uncertainties, i.e. the uncertainties of obliquity of the ecliptic and efpuinox and the inaccurary of lunar love numbers adopted. At present, with exception of the corrections of ocean tide and pole tide the theoretical modeling such as earth solid tide, lunar tide, plate motion and the relativity modeling, especially the space curvature cansed by the earth, have been considered to match the present observing precision in the magnitude of centimeter. The equivalent of relativity modeling used in geocentric or barycentric reference frame is further described.
\end{abstract}

\section{INTRODUCTION}

Since the emplacement of retroreflectors on the lunar surface by Apollo astronants in Angust 1069, laser ranging to the moon has been carried out for more than 20 years. Now the project of Lunar Laser Ranging(LLR) is being carried out at McDonald, USA(MICD) and CERGA, France (CER). Haleakala station, Hawaii, USA(HAL) stopped this project in 1990 clue to a lack of funds from NASA. Satellites such as Lageos, Etalon, Starlette etc. are observed routinely and the attempts of LLR are made at Wettzell, Germany and Orroral station,Australia. One meter telescope was constructed by Communication Research Laboratory(CRL) in Japan where to range the reflectors on the moon can be done frequently. The pointing precision of 1.2 meter telescope attained to $2-3^{\prime \prime}$ and part of hardware for LLR was availalble at Yunum Observatory, China. The present precisions of normal points (Jin et al. 1092) attain to $5 \mathrm{~cm}$ due to the technical improvements such as reduction of pulse width from $2.5 \mathrm{~ns}$ to $100 \mathrm{ps}$, use of micro channel plate etc.. There are five LLR clata analysis centers in the International Earth Rotation Service(IERS), i.e. JPL, USA, CERGA, France, SHA, China, UTXMO, USA and Munich University, Germany.

The theoretical modeling of LLR data reduction is gradually perfected at Shanghai Observatory. Two lunar and planetary ephemerides were used in our work.
(A) before 1989
DE200/LE200
(B) in 1989
DE200 and lunar physical
(C) after 1080
libration of Eckliardt 500
DE303/LE303 
The reason why physical libration of Eckhardt 500 was used is that in March 1089 the libration tape LE200 was ended to use. The pre-residual and post residual of using different lunar and planetary ephemerides during the periud of January 108t to March 1989 are shown in Fig.1. There is large systematic difference by use of DE200 and the lunar physical libration of Eckhardt 500 in the pre-residuals because these are not procluced by numerical integration of the equation of motions simultaneonsly. After arljustment of fourty parameters such as the coordinates of stations and reflectors, orbital elements of the moon and the earth, linar moment of inertia ratios $\beta$ and $\gamma$, coefficients of the thirddegree harmonics in the lunar gravitational potential etc., the root mean square(rms) of post-resirlual should equal to that of normal point. As shown in Fig.1(b) the rms of postresichal is three or four times larger than the observing precision becanse of the innerfect theoretical model, uncertainties of ephemeris, the correlation between 40 clements of matrix and so on. It will be cliscussed in detail in the following section.

\section{EPHMERIS UNCERTAINTIES}

The model errors of propagation, refraction, solid earth ticle, lunar tide and plate motion are almost less than $1 \mathrm{~cm}$, but the lunar love numbers vary with various lunar models to a greater extent and it equals to the value itself as shown in Table 1.

Table 1. Different Lunar Love Numbers of Various Lumar Models

\begin{tabular}{|c|c|c|c|c|c|c|}
\hline Author & \multicolumn{2}{|c|}{$\begin{array}{c}\text { Cheng and Toksez } \\
(198 \pi)\end{array}$} & $\begin{array}{c}\text { Dazliang Han } \\
(1084)\end{array}$ & $\begin{array}{l}\text { Bodri } \\
(1986)\end{array}$ & \multicolumn{2}{|c|}{$\begin{array}{c}\text { C'hengzhin Zhang } \\
(1090)\end{array}$} \\
\hline Model & \multicolumn{2}{|c|}{$\begin{array}{l}\text { homogeneous and } \\
\text { two layers model }\end{array}$} & Bills and Ferrar & \multicolumn{3}{|c|}{$\begin{array}{l}\text { unlomogeneous donsity } \\
\text { and compressible }\end{array}$} \\
\hline & A & B & & & luna $89-(01$ & luna $89-(02$ \\
\hline$h_{2}$ & 0.0501 & 0.0627 & 0.0929 & 0.0503 & 0.04225 & 0.04676 \\
\hline$k_{2}$ & 0.0293 & 0.0335 & 0.0535 & 0.0281 & 0.02425 & 0.112680 \\
\hline$l_{2}$ & 0.0198 & 0.0240 & 0.0205 & 0.0133 & 0.01214 & 0.01236 \\
\hline
\end{tabular}

The influence of lunar love numbers npon the distance from obscrving station to reffector was discussed in the poster paper of this meeting. When the moon was near perigee on September $6^{d} .23526 \mathrm{UT}, 198 \mathrm{t} \Delta \rho_{2}$ is $16.5 \mathrm{~cm}$ and $15.8 \mathrm{~cm}$ for reflector A14 and A15, while near the apogee of the moon on September $15^{d} .55072 \mathrm{UT}, 108 \mathrm{~T}$ it is $11.3 \mathrm{~cm}$ and $7.5 \mathrm{~cm}$ respectively. The average value is $12.8 \mathrm{~cm}$.

Becanse the lunar laser ranging is sensitive to the clirection of pole of the earth's rotation, i.e. sensitive to the of-clate celestial equator and also sensitive to the of-clate ecliptic from the solar perturbation upon the lunar orbit, the variation of theoretical distance from the station to the retroreflector on the moon is cansed by the uncertainties of oblicpuity of the ecliptic $\Delta \varepsilon$ and that of equinox $\Delta E$ and is expressed as the following formula.

$$
\left\{\begin{array}{l}
\Delta \rho_{1}=\bar{a}\left[(\cos \bar{\phi} \cos \bar{t} \sin \bar{\delta}-\sin \bar{\phi} \cos \bar{\delta})^{2}(\Delta \delta)^{2}+(\cos \bar{\phi} \cos \bar{\delta} \sin \bar{t})^{2}\right]^{1 / 2} \\
(\Delta \bar{t})^{2}=(\Delta \bar{\pi})^{2}=(\Delta E)^{2} \\
\Delta \bar{\gamma} \cos \bar{\delta}=(\cos \bar{\beta} \sin \bar{\lambda} \cos \epsilon-\sin \bar{\beta} \sin \epsilon) \Delta \epsilon
\end{array}\right.
$$

where $\bar{a}$ and $\bar{\phi}$ denote the geocentric distance and latitude of observing station; $\bar{t}, \bar{\pi}$ and $\bar{\delta}$ inclicate low angle, right ascension and declination of retroreftector; $\bar{\lambda}$ and $\bar{\beta}$ indicate the longitude and latitude of reflector; $\epsilon$ denote the obliquity of the ecliptic and $\Delta \bar{t}$ and $\Delta \bar{x}$ denute the nncertainty of hour angle and right ascension of reflector. For example, 
the variation of distance was calculated at $12^{h}$ UT every day of March 1087 and is shown in Fig.2 , by using formula (1) in which $\pi$ is adopted as the average value of contatorial radins of the earth. the coordinates of lunar center is used instead of the coordinates of reflectors for calculation .hour angles are adopted as $\pm 0^{h}$ and $\pm 3^{h}$ respectively and $\Delta E$ and $\Delta E$ are $\pm 0 . " 01$ at latitude of $20^{\circ} .30^{\circ}$ and $40^{\circ}$. In Fig. 2 it is shown that $\Delta \rho$ induced by the nucertainties of cphemeris does not change too much with latitude while it varies with hour angle to a great extent.

In practical calculation $\Delta \varepsilon$ and $\Delta E$ are $\pm 0 .{ }^{\prime \prime} 01$ and $\pm 0 . " 02$ which include the error of the precession constant about 0."1/cty (Standish 1987). For Mr.Donald Observatory the maximum value of $\left(\Delta \rho_{1}\right)_{\max }$ is $41.4 \mathrm{~cm}$ induced by $\Delta \varepsilon$ and $\Delta E$ if $\mathrm{t}=45^{\circ}, \lambda=-90^{\circ}$ and $\delta=e-i$ where $\mathrm{i}$ is the inclination of lunar orbit to the ecliptic while $\left(\Delta \rho_{1}\right)_{a}=33.6 \mathrm{~cm}$ at $5^{h} .3844$ UT on March 9. 1987. It is clear that the influence of ephemeris uncertainty on the distance from the observing station to reflector is 2.5 times larger than that of different lunar love numbers adopted.

Status of the precision of LLR data obtained from 1970 to 1987 is shown in Talble 2(Nic et al. 1991). In this period DE200/LE200 was used for LLR data analysis at Shanghai Observatory.

Table 2. Precision of LLR unit: cm

\begin{tabular}{lrrrrr}
\hline Station & MCD & CER & MLRS & HAL & Average \\
\hline $\mathrm{N}$ & 3338 & 1548 & 620 & 480 & \\
$\Delta \rho_{o b}$ & $13.7 \mathrm{~T}$ & 19.41 & 7.06 & 4.33 & 13.78 \\
$\Delta \rho_{\text {pre }}$ & 51.41 & 58.41 & 55.57 & 49.53 & 53.63 \\
$\Delta \rho_{\text {post }}$ & 46.07 & 44.69 & 33.72 & 23.48 & 42.62 \\
\hline
\end{tabular}

where $N, \Delta \rho_{o b} . \Delta \rho_{p r e}$ and $\Delta \rho_{p o s t}$ denote the number of normal point, root mean square (rms) of normal point, of pre-residual and of post-residual.

$$
\begin{aligned}
& \bar{m}=\left(\Delta \rho_{p r e}^{2}-\Delta \rho_{c b}^{2}\right)^{1 / 2} \\
& \bar{n}=\left(\Delta \rho_{p o s t}^{2}-\Delta \rho_{o b}^{2}\right)^{1 / 2}
\end{aligned}
$$

From Table $2 \bar{m}$ and $\bar{n}$ can be calculated with formula (2) for all stations and the results are $51.83 \mathrm{~cm}$ and $40.33 \mathrm{~cm}$ respectively. For MICD station the corresponding values of $\mathrm{m}$ and $\mathrm{n}$ are $49.53 \mathrm{~cm}$ and $43.96 \mathrm{~cm}$.

If the main source of error is cansed by the orientation error of ephemeris reference frame and the inaccurary of lunar love numbers, the sum of the maximum error is expressed as follows

$$
\Delta \bar{\rho}_{\max }=\left[\left(\Delta \bar{\rho}_{1}^{2}\right)_{\max }+\left(\Delta \bar{\rho}_{2}^{2}\right)_{\max }\right]^{1 / 2}
$$

The value of $\Delta \bar{\rho}_{\text {max }}$ should be consistent with the following condition:

$$
\bar{n} \leq \Delta \bar{\rho}_{\max }<\bar{m}
$$

For general situation, $(\Delta \bar{\rho})_{a}=\left[\left(\Delta \bar{\rho}_{1}\right)_{a}^{2}+\left(\Delta \bar{\rho}_{2}\right)_{a}^{2}\right]^{1 / 2}<\bar{n}$. The data from Mcdlanald observatory is $56 \%$ of all LLR data, so

$$
\text { and } n \leq \Delta \rho_{\max } \leq m, \Delta \rho_{a}<n
$$

According to the previous analysis $\left(\Delta \rho_{1}\right)_{\max }=41.4 \mathrm{~cm},\left(\Delta \rho_{1}\right)_{a}=33.6 \mathrm{~cm} .\left(\Delta \rho_{2}\right)_{\text {max }}=$ $16.5 \mathrm{~cm}$ and $\left(\Delta \rho_{2}\right)_{a}=12.8 \mathrm{~cm}$, so $(\Delta \rho)_{\max }=44.6 \mathrm{~cm}$ and $\Delta \rho_{a}=30.0 \mathrm{~cm}$ shonld be ol)tained. These results are consistent with analysis mentioned above so the theoretical analysis concerned the error of the post-residual is correct. Althongh IERS recommended 
that DE200/LE200 should be used in analysis of SLR and VLBI, in LLR the parameters of earth-moon system should be fitted or a more recent lunar ephemeris should be used. Becanse of the uncertainties of ephemeris and the correlation between elements of matrix we have our preference to adopt later suggestion of IERS.

\section{RELATIVITY MODELING IN LLR DATA ANALYSIS}

Our software of reducing the LLR data has gradually perfected for the relativity model. Recently the space curvature of earth term and Lorentz contraction (Xu et al. 1993, in press) was added. In the reduction of LLR data three kinds of relativety corrections should be considered.

\subsection{Transformation of Epoch and Time Interval}

The epoch and time interval of a range to the moon are recorded at the station in UTC. Since the reference frame workshop of IAU was established, the concept of time has been discussed. For the barycentric and geocentric reference frame now the TC.B and TCG are suggested to be used. Because the time argument of the planetary cphemeris of DE200 or DE303 is TDD, observing epoch UTC will be transformed into TDB for reduction of LLR data. There are two formulae to transform TDT to TDB, i.e. Moyer's and Fairheall's. The precision of using two formula are 1 H.s and 100 us respectively. Dr. Fairhead (Fairhead 1987) gave the more precise formula than Moyer did, but Moyer's formula has matched the present observing precision of LLR and is still used with the overlap method to obtain the range epoch in solar barycentric system.

\subsection{Spatial Coordinates Transformation}

Because of the computation of LLR data in solar barycentric system the positional vector $\vec{r}$ of station should be transformed from geocentric system into barycentric system. The tranformation factor of scale is abont $15 \mathrm{~cm}$ in the height of tracking station and the Lorentz contraction is about $3 \mathrm{~cm}$ for station coordinates. The sclenocentric coordinate of reflector should be transformed first into geocentric frame. This corrected value is less than $3 \times 10^{-5} \mathrm{~m}$, which can completly be nogeleted. Then, the scale effect is 4 cm and the maximum value of Lorentz contraction effect (from special relativity) is about $1 \mathrm{~cm}$ for reflector coordinates to be transformed from geocentric system to barycentric system. Since the maximum influence of the moon and the major planet for $\Psi$, the geocentric gravitational potential, is about $2 \times 10^{-5} \mathrm{~m}$, only the solar term should be considered.

\subsection{Space Curvature}

Due to the gravitational influence of the sun, the earth and the mon the space curvature in different level of neighbouring space exist. As for this reason the time interval of pulse between the station and the retroreflector is increased. The gravitational time delay induced by the Sun, Earth, Moon and Jupiter are listed in Talble 2.

Talble 2. Estimation of Gravitational Time Delay for LLR in Two-way 


\begin{tabular}{ccccc}
\hline gravitational body & Sun & Earth & Moon & Jupiter \\
\hline$\Delta t_{\text {grav }}$ & $5 \times 10^{-6} \mathrm{~s}$ & $2 \times 10^{-10} \mathrm{~s}$ & $3 \times 10^{-12} \mathrm{~s}$ & $1 \times 10^{-11} \mathrm{~s}$ \\
c $\Delta t_{\text {grav }}$ & $16 \mathrm{~m}$ & $7 \mathrm{~cm}$ & $1 \mathrm{~mm}$ & $4 \mathrm{~mm}$ \\
\hline
\end{tabular}

To match the present observing precision in the magnitude of centimeter the space curvature caused by the earth should be considered. After improving the precision of LLR to millimeter the other terms such as the gravitational time delay induced by the Moon and the Inpiter should be considered too.

Dr. Han theoretically proved the equivalent of SLR or LLR reduction in solar byrycenter and geocentric reference system with general relativistic theory (Han 1990). This point was confirmed in processing SLR observational data(Hunag 1990). Obviously the obscrvation of LLR is in geocentric reference. If the lunar and planetary epheremis in geocentric reference frame are provided, it will be convenient for processing LLR data in geocentric frame.

\section{CONCLUSION}

4.1. The uncertainty of epheremis is main error source of present rednction of LLR. To use the more recent lunar and planetary ephemeris for LLR data processing is suggested.

4.2. To match the present olsserving precision ( in magnitude of centimeter) the effect of space curvature from the earth should be considered.

4.3. Reduction of LLR data in the solar system is equivalent to that in geocentric system. According to the planteary ephermeris in solar barycentric or geocentric: system the reference frame of LLR data reduction will be chosen.

\section{ACKNOWLEDGEMENTS}

We thank the geodetic group of Jet Propulsion Laboratory, USA for their kindness to provide us lunar and planetary ephemericles and thank Dr. C. Veillet, C.ERGA, France for his kind offer of the global data of LLR too.

\section{REFERENCE}

1. Fairhead,L. (1987) Proceeding of IAU Symposium No.128 p.419-426.

2. Han, C.H.(1090) ' Geocentric and Celestial Reference Frame in General Relativity Frame', Thesis of PhD Degree, Zhengzhou Institute of Surveying and Mapping, China.

3. Hunag, C.(1990) Celestial Mechanics and Dynamical Astronomy Vol. 48 p.167-185.

4. Jin, W.J.(1092) Acta Astrophysics Vol. 12 No. 2 p.133-141.

5. Nie,Z.M.(1991) Acta Astronumica Sinica. Vol. 32 No. 4 p.402-408.

6. Standish,E.M. Jr.(108T) Proceedings of IAU Symposium No.128, p.49-54.

7. Xu,H.G.ct al.(1093) Annals Shanghai Olsservatory Academia Sinica No.14 (in press). 


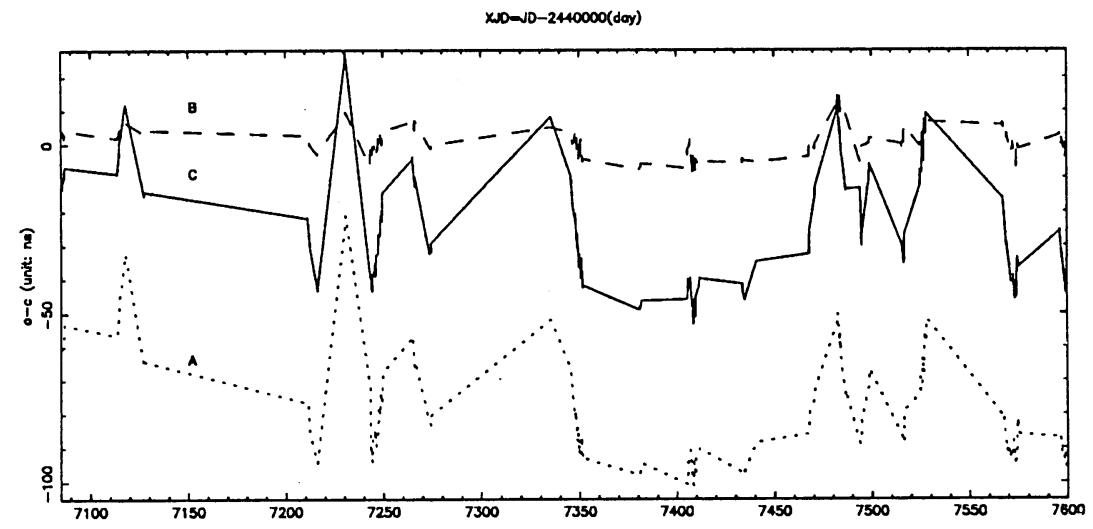

Fig.1(a) Pre-residual of reflector No.3 observed at CER station

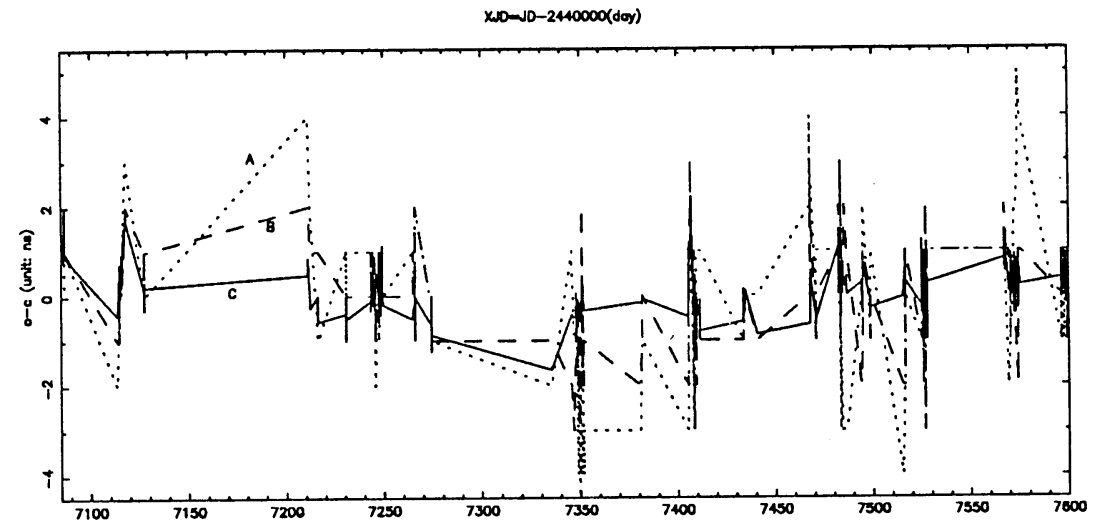

Fig.l(b) Post-residual of reflector No.3 observed at CER station
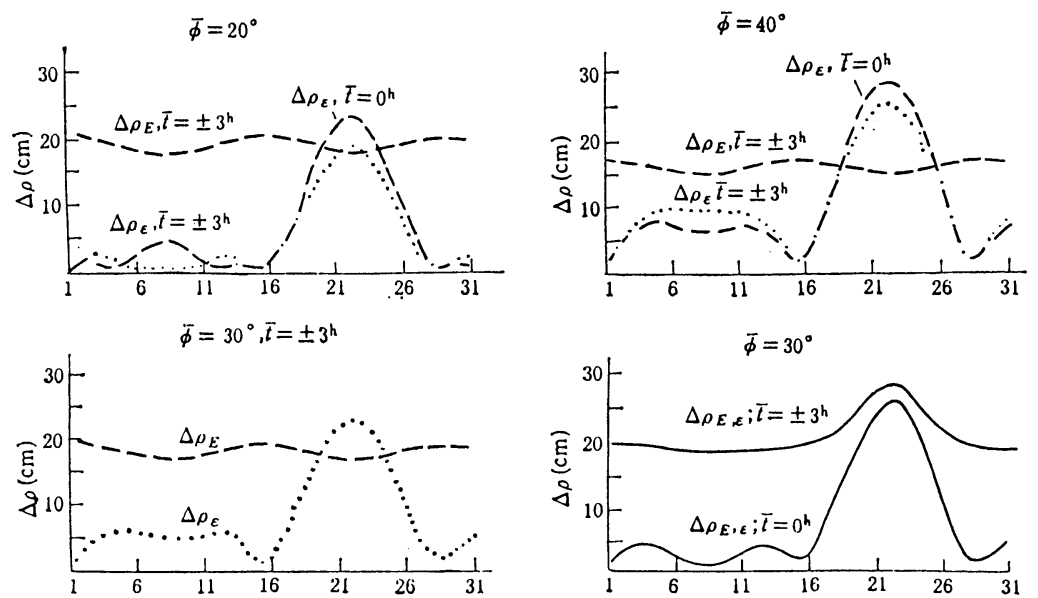

Fig.2 Ilistory of the distance errors resulting from the uncertainties of the eplicmeris

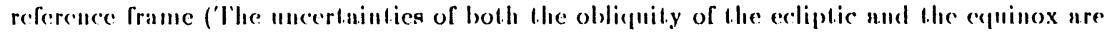
\pm 0 ". 01 and the curves of the distance errors dependent on every day of Mlarch, 198T are also givell) 